\title{
O sistema bancário brasileiro: Uma análise do desempenho através da DEA
}

\author{
Ana Carolina Thomaz de Almeida Monteiro Barbosa \\ Graduanda em Administração pela Universidade Federal Rural do Rio de Janeiro - \\ UFRRJ \\ Rod. BR 465 - Km 07. Seropedica/RJ. CEP: 23890-000 \\ E-mail: acbarbosa@ufrrj.br \\ Marcelo Alvaro da Silva Macedo \\ Doutor em Engenharia de Produção pela Universidade Federal do Rio de Janeiro- \\ UFRJ \\ Professor da Universidade Federal Rural do Rio de Janeiro - UFRRJ \\ Rod BR 465 - Km 07. Seropedica/RJ. CEP: 23890-000 \\ E-mail: alvaro@ufrrj.br
}

\section{RESUMO}

Este artigo tem como objetivo a avaliação da eficiência do mercado bancário brasileiro por meio de uma análise evolutiva (2001-2007) dos bancos em atividade no país. Para tanto, com base nos dados extraídos do Relatório dos 50 Maiores Bancos publicado pelo Banco Central do Brasil, sugeriu-se a utilização da metodologia DEA para avaliação do desempenho dos bancos públicos e privados, sejam eles de controle nacional ou estrangeiro. Os resultados revelaram a existência de algumas instituições 100\% eficientes, que possuem uma excelente combinação de recursos de entrada e saída, não necessitando de melhorias. Além disso, observou-se não existir relação entre eficiência e tamanho nas análises dos segmentos. Isso mostra que, nestes ramos, não há vantagem dos bancos de grande porte em relação aos médios e pequenos, ou seja, que não é o tamanho, mas sim o desempenho que gera a competitividade.

Palavras-chave: Desempenho. Bancos. DEA.

\section{The Brazilian Banking System: A performance analysis through the DEA}

\begin{abstract}
This article aims to measuring the efficiency of the Brazilian banking market through a temporal analysis (2001-2007) of banks in activity in the country. To this end, based on data extracted from the Report of the 50 largest banks of $B C$, it is suggested to use the DEA methodology for assessing the performance of public and private banks, whether of domestic or foreign control. The results showed there some institutions $100 \%$ efficient, they have an excellent combination of inputs and outputs, without the need for improvements. Moreover, there is no correlation between size and efficiency in the analysis of the segments. This shows that in these segments, there is no advantage of large banks in relation to medium and small, that is not the size, but the performance that creates competitiveness.
\end{abstract}

Key Words: Performance. Banks. DEA. 


\section{INTRODUÇÃO}

A indústria bancária, de maneira geral, possui um papel fundamental na economia mundial. Como principal agente financiador das instituições, torna-se indispensável que os bancos utilizem ferramentas confiáveis de mensuração da performance como mecanismo de gestão.

Porém, não existe um modelo de avaliação de desempenho organizacional que seja único para todas as variáveis do mundo empresarial. No entanto, alguns métodos são capazes de considerar aspectos diversificados, assumindo, assim, um papel crucial na avaliação da performance empresarial, como a medida de eficiência DEA (Data Envelopment Analysis ou Análise Envoltória de Dados), utilizada neste trabalho.

A resposta mais importante desta metodologia é a caracterização de uma medida de eficiência, que faz com que a decisão fique orientada por um único indicador construído a partir de várias abordagens de desempenho diferentes. Vale ressaltar que isso facilita o processo decisório, pois, ao invés de considerar vários índices para concluir a respeito do desempenho da empresa ou da unidade sob análise, o gestor se utiliza apenas da medida de eficiência da DEA. Além disso, a metodologia DEA mune a alta administração com informações adicionais sobre os maiores determinantes de eficiência ou ineficiência, partindo- se de variáveis pré-selecionadas por meio de uma análise de benchmarking, com a qual o gestor pode avaliar as alterações necessárias para que a instituição possa se tornar eficiente em termos competitivos.

Nesse contexto, este trabalho se propõe a avaliar o desempenho das maiores instituições bancárias, segundo relatório do Banco Central do Brasil (Bacen), no período 2001-2007, utilizando a Análise Envoltória de Dados como metodologia de análise. Para tanto, um modelo de análise foi construído levando em consideração o nível de uso dos principais insumos (instalações físicas gerais, pessoal e postos de atendimento) e a lucratividade de cada banco ao longo do período sob análise.

\section{AVALIAÇÃO DE DESEMPENHO}


A globalização e a abertura dos mercados fizeram com que as organizações sofressem mudanças para se adaptarem ao novo quadro mundial. Neste quadro, em que a competitividade é um fator determinante, os sistemas de medição de desempenho se tornaram indispensáveis.

A mensuração de desempenho é definida por Neely (1998) como um processo que visa a quantificação da eficiência e a da efetividade de ações passadas, levando em conta a aquisição, coleta, classificação, análise, interpretação e disseminação dos dados apropriados. Ao final desta trajetória, os sistemas de medição fornecem o grau de evolução, estagnação ou involução dos processos e atividades das empresas sob análise, gerando informações importantes e a tempo de permitir que ações preventivas e/ou corretivas sejam tomadas. Como o progresso da empresa é monitorado por meio destes sistemas, seus eventuais erros são corrigidos, aumentando as possibilidades de que estas conquistem suas metas e objetivos.

Os processos de mensuração de desempenho se desenvolvem a partir da seleção de indicadores críticos de desempenho específicos para cada empresa. Entretanto, a maior dificuldade dos gestores está na escolha desses indicadores. $O$ principal meio de defini-los seria, primeiramente, identificar onde a organização se encontra e aonde quer chegar, para posteriormente definir os mecanismos que farão com que isso seja possível. Tais processos são permanentes e repetitivos, sendo a frequência de suas medições dependente da atividade a ser medida.

Para Slack et al. (2002), toda organização precisa, de alguma forma, de medidas de desempenho como um pré-requisito para melhoramento. No entanto, as práticas de medição de desempenho variam entre as empresas. Cabe ressaltar, porém, que depois da mensuração de desempenho, usando, normalmente, um conjunto de medidas parciais, a organização precisa fazer um julgamento sobre sua performance. Este julgamento, segundo os autores, envolve a comparação do nível de desempenho atingido com algum tipo de padrão. Esses padrões podem ser: 
- Padrões Históricos: consistem na comparação do desempenho atual com desempenhos anteriores, construindo assim uma tendência do comportamento de desempenho ao longo do tempo;

- Padrões de Desempenho Alvos: são aqueles estabelecidos arbitrariamente para refletir algum nível de desempenho que é visto como adequado;

- Padrões de Desempenho da Concorrência: utilizam a comparação do desempenho atingido com aquele que está sendo conseguido por um ou mais competidores;

- Padrões de Desempenho Absolutos: são aqueles tomados em seus limites (ex.: estoque zero, zero defeito, etc.).

Quando as empresas utilizam o mercado como parâmetro de comparação, segundo padrões de desempenho da concorrência, faz-se necessário usar uma abordagem denominada benchmarking. Essa abordagem consiste na performance relativa das empresas em função de um conjunto de competidores previamente selecionados (SLACK et al., 2002).

Camp (1989) define benchmarking como sendo um processo sistemático para a identificação da melhor prática e para a modificação do conhecimento existente, de modo a alcançar um desempenho superior. Para Novaes (2001), benchmarking é considerado um processo contínuo e sistemático executado através da comparação entre as unidades sob análise, que leva ao estabelecimento de ações gerenciais efetivas, com o objetivo de aprimorar os resultados (redução de custos, aumento na produção, etc.); além de ser um dos modernos instrumentos de gerência, que possibilita a melhoria do desempenho técnico-econômico das empresas de forma comparativa ou relativa.

Os sistemas de mensuração de desempenho são essenciais para que as organizações possam competir no mercado global. Porém, as avaliações feitas em caráter relativo, ou seja, mensurando a eficiência da organização em relação, por exemplo, ao seu ambiente competitivo, são geradoras de resultados potencialmente 
consistentes. Isto ocorre à medida que informam o posicionamento da empresa perante a referência e as demais empresas.

Não existe a possibilidade de reduzir a complexidade do desempenho de um negócio a um único indicador, havendo necessidade real de aplicar diversos indicadores para atingir os mais variados aspectos em que a estratégia de negócio se realiza (SLACK et al., 2002). Assim, um programa de avaliação de desempenho eficiente deve incluir todos esses elementos considerados críticos de sucesso. Isto quer dizer que todos os elementos relevantes às empresas, de maneira individual, precisam ser, de alguma forma, mensurados para permitir análises comparativas.

São inúmeras as variáveis do mundo empresarial passíveis de mensuração. No entanto, não existem, ainda, modelos de avaliação de performance únicos para todas estas variáveis. A solução encontrada pelos gestores é a utilização de uma série de metodologias de avaliação capazes de tratar os diferentes elementos da organização. Essas técnicas produzem resultados isolados, uma vez que não consideram a multiplicidade de fatores numa única análise.

Neste sentido, a Análise Envoltória de Dados (DEA) apresenta-se como uma técnica que gera uma medida de desempenho capaz de comparar a eficiência de várias unidades operacionais similares mediante a consideração explícita do uso de suas múltiplas entradas para a produção de múltiplas saídas. Desta forma, esta metodologia faz com que a decisão fique orientada por um único indicador relativo, construído a partir de várias abordagens de desempenho diferentes. Portanto, como congrega diversas perspectivas, a técnica promove uma melhor percepção da performance organizacional.

\section{ANÁLISE ENVOLTÓRIA DE DADOS (DEA)}

Para Silva, Neves e Gonçalves Neto (2003), a metodologia DEA, que teve origem no trabalho de Farrell (1957), caracteriza-se como uma técnica não paramétrica que permite lidar com variáveis de saída (outputs) e entrada (inputs), com o objetivo de analisar, comparativamente, unidades independentes no que se refere ao seu 
desempenho, ou seja, à eficiência de cada unidade.

São várias as formulações dos modelos de DEA encontradas na literatura, conforme mencionam Charnes et al. (1994), entretanto dois modelos básicos são geralmente usados nas aplicações. O primeiro modelo chamado de CCR (CHARNES, COOPER e RHODES, 1978), também conhecido como CRS (Constant Returns to Scale), avalia a eficiência total, identifica as DMU's eficientes e ineficientes, e determina a distância em que as unidades ineficientes da fronteira se encontram da eficiência. O segundo modelo denominado de modelo BCC (BANKER, CHARNES e COOPER, 1984), também conhecido como VRS (Variable Returns to Scale), utiliza uma formulação que permite a projeção de cada DMU ineficiente sobre a superfície de fronteira (envoltória) determinada pelas DMU's eficientes de tamanho compatível.

No caso das formulações, além das da escolha entre CRS e VRS, existe a necessidade de fixação da ótica de análise (orientação input ou orientação output). Macedo et al. (2006a) assinalam que a abordagem DEA baseada nas entradas (inputs) busca maximizar as quantidades de produtos, isto é, maximizar uma combinação linear das quantidades dos vários produtos da empresa. Já para uma abordagem baseada nas saídas (outputs), busca-se minimizar as quantidades de insumos, isto é, minimizar uma combinação linear das quantidades dos vários insumos da empresa.

Um caminho intuitivo para introduzir DEA, segundo Lins e Meza (2000), é por meio de forma de razão. Para cada DMU procura-se obter uma medida de razão de todos os outputs sobre todos os inputs ou ainda de todos os inputs sobre todos os outputs. Ou seja, a modelagem procura encontrar os pesos ótimos uj e vi, para a resolução de um dos seguintes problemas de programação matemática:

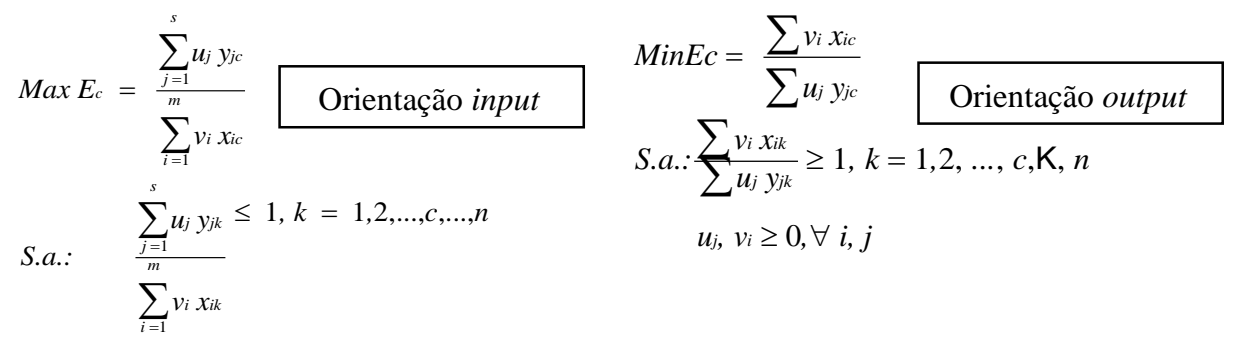


No modelo com orientação input, c é a unidade (DMU) que está sendo avaliada. O problema acima envolve a procura de valores para u e $\mathrm{v}$, que são os pesos, de modo que maximize a soma ponderada dos outputs (output "virtual") dividida pela soma ponderada dos inputs (input "virtual") da DMU em estudo, sujeita à restrição de que esse quociente seja menor ou igual a um para todas as DMU's. Esta função está sujeita à restrição de que, quando o mesmo conjunto de coeficientes de entrada e saída (os vários vi e uj) for aplicado a todas as outras unidades que estão sendo comparadas, nenhuma unidade excederá $100 \%$ de eficiência ou uma razão de 1,00.

Já no modelo com orientação output, a eficiência é calculada pelo inverso da função objetivo, ou seja, eficiência $=1 / E$. Este problema define a relação dos inputs sobre os outputs, onde c é o índice da unidade que está sendo avaliada. Tem-se neste problema as mesmas variáveis de decisão uj e vi, porém, se quer minimizar a soma ponderada dos inputs ("input" virtual) dividida pela soma ponderada dos outputs ("output" virtual) da DMU em estudo, sujeita a restrição de que este quociente seja maior ou igual a um, para todas as DMU's.

Segundo Coelli, Rao e Baltese (1998), este é um problema fracionário (não linear) de programação matemática de difícil solução, que pode ser facilmente resolvido transformando a relação em uma função linear, simplesmente considerando o denominador da função objetivo igual a um. De acordo com Charnes et al. (1994), os modelos DEA-CRS-I e DEA-CRS-O podem, então, ser apresentados da seguinte maneira:

$$
\begin{aligned}
& \operatorname{Max} E_{c}=\sum_{j=1}^{s} u_{j} y_{j c} \\
& \text { S.a.: } \quad \sum_{i=1}^{m} v_{i} x_{i c}=1 \\
& \operatorname{Min} \sum_{i=1}^{m} v_{i} x_{i c} \\
& \text { S.a. }: \sum_{j=1}^{s} u_{j} y_{j c}=1 \\
& \sum_{j=1}^{s} u_{j} y_{j k}-\sum_{i=1}^{m} v_{i} x_{i k} \leq 0, k=1,2, \ldots, c, \ldots, n \\
& \sum_{i=1}^{m} v_{i} x_{i k}-\sum_{j=1}^{s} u_{j} y_{j k} \geq 0, k=1, \mathbf{K}, c, \mathbf{K}, n \\
& u_{j}, v_{i} \geq 0, \forall i, j . \\
& u_{j}, v_{i} \geq 0, \forall i, j
\end{aligned}
$$


Estas formas do problema são conhecidas como problemas dos multiplicadores, como também são chamados os pesos, uj e vi. Macedo et al. (2006a) argumentam que no modelo com orientação input, quanto maior a relação $\square$ outputs / $\square$ inputs maior a eficiência. Para cada DMU a ser analisada, formula-se um problema de otimização com o objetivo de determinar os valores que essa DMU atribuiria aos multiplicadores u e v de modo a aparecer com a maior eficiência possível. Então, complementam os autores, o problema consiste em achar os valores das variáveis uj e vi, que são os pesos (importância relativa de cada variável/critério), de modo que se maximize a soma ponderada dos outputs (output "virtual") dividida pela soma ponderada dos inputs (input "virtual") da DMU em estudo, sujeita a restrição de que esse quociente seja menor ou igual a um, para todas as DMUs.

Já no modelo com orientação output, continuam os autores, quanto menor a relação inputs / outputs maior a eficiência. Para cada DMU a ser analisada, formula-se um problema de otimização com o objetivo de determinar os valores que esta DMU atribuiria aos multiplicadores uj e vi de modo a aparecer com a maior eficiência possível, ou seja, minimizando a soma ponderada dos inputs (input "virtual") dividida pela soma ponderada dos outputs (output "virtual") da DMU em estudo, sujeita a restrição de que esse quociente seja maior ou igual a um, para todas as DMUs.

\section{O MERCADO BANCÁRIO BRASILEIRO: UMA ANÁLISE INTRODUTÓRIA}

A elevada inflação, que marcou a economia brasileira de 1960 à 1994, proporcionou às instituições bancárias, durante todos estes anos, possibilidades de ganhos extraordinários por aplicações financeiras.

Entretanto, em 1994, a implantação do Plano Real, trazendo um novo ambiente de estabilidade inflacionária, levou muitos bancos a perderem esses ganhos, sendo obrigados a buscar novas fontes de receita, fundamentadas em resultados operacionais, tais como o aumento na cobrança de tarifas pela prestação de seus serviços. 
Só oito anos depois, em 2002, a estabilidade se fez realmente sentir com os bancos apresentando os maiores índices de rentabilidade média, desde o início do plano.

Em 2003, os resultados de todo o setor financeiro foram prejudicados em função de uma queda nas cotações do dólar. Entretanto, esta queda não proporcionou efeito generalizado sobre as instituições bancárias, líderes dos grandes conglomerados financeiros. Em muitos casos, inclusive, os resultados destas instituições superaram os de 2002.

Neste mesmo momento, o processo de concentração do sistema financeiro, já iniciado há alguns anos, como resposta a estabilidade econômica e a abertura comercial num contexto de intensa competitividade, teve continuidade com a manutenção da política de aquisição de instituições financeiras menores como alternativa de sobrevivência.

O ano seguinte -2004- caracterizou-se também por excelentes resultados, que se fundamentaram na diversificação das receitas, o que orientou o crescimento não só por operações de crédito (microcrédito e crédito consignado) e tesouraria como, principalmente, por serviços. Verificam-se, porém, elevados gastos administrativos e com pessoal debilitando, de certa forma, os ganhos destas instituições.

Em 2005, tem-se, em relação ao período anterior, um crescimento do resultado líquido em torno de $40 \%$. Na realidade, este percentual reflete a nova fase em que 0 sistema bancário se encontrava, baseada em uma diminuição significativa das despesas administrativas e de pessoal, em investimentos em TI, na manutenção de investimentos em serviços e, em especial, na busca por uma estrutura enxuta e mais eficiente, capaz de permitir a adoção de uma postura agressiva.

Em 2006, pela primeira vez, as instituições bancárias enfrentaram um corte contínuo dos juros básicos e, ainda, uma corrida em busca de clientes que as obrigou a reduzir os juros cobrados em seus empréstimos. Da mesma forma que em 2005, o crédito foi estimulado, porém, em linhas de pessoas físicas, onde o aumento do salário real e do emprego teve alcance. O preço por esta opção foi a elevação das taxas de 
inadimplência. O período se beneficiou, além disso, com a ascensão do crédito imobiliário, verificada até os dias atuais.

\section{DESEMPENHO ORGANIZACIONAL NO SETOR BANCÁRIO}

A partir da década de 1980 e com maior intensidade na década de 1990, podese observar a Análise Envoltória de Dados como um instrumento de crescente utilização para análises de eficiência em instituições bancárias. Segundo Mostafa (2007), foram encontrados 130 trabalhos de análise de eficiência bancária sob a abordagem da DEA, sendo 116 publicados apenas entre 1992 e 1997. Nesta seção são descritos, brevemente, alguns trabalhos que possuem a DEA como metodologia de avaliação.

Barr et al. (2002) avaliaram o desempenho, em caráter relativo de alguns bancos comerciais americanos no período de 1984 a 1998, utilizando a metodologia DEA CRS, orientação input, aplicada a dados provenientes de demonstrações contábilfinanceiras. Os resultados revelaram existirem fortes e consistentes relações entre eficiência e algumas medidas independentes de desempenho. Segundo os autores, o modelo se mostrou extremamente útil para os bancos porque estabelecia um ponto de referência - benchmarking - com outras instituições, atuando como uma ferramenta de vigilância complementar no processo de verificação de desempenho.

Assim como os autores supracitados, Mostafa (2007) também se utilizou da análise de benchmarking gerada pela metodologia. Dessa forma, através da DEA VRS, orientação output, o pesquisador mensurou a eficiência de 85 , dentre os 100 bancos árabes, classificados como maiores no ano de 2005. Os resultados revelaram oito bancos como eficientes, sendo estes referenciais de boas práticas para os demais. Notou-se, porém, que apenas um destes bancos se sobressaiu no número de vezes em que apareceu como benchmarking.

Macedo et al. (2006b) analisaram, em seu trabalho, os maiores bancos em atividade no Brasil nos anos de 2001 a 2004, segundo a Revista Valor Financeiro, com 
a finalidade de formar um painel de análise da competição no setor bancário brasileiro. A metodologia utilizada pelos autores foi a DEA - CRS com uso de fronteira invertida, orientada tanto para input, quanto para output. As variáveis de saída consideradas foram: liquidez imediata e rentabilidade operacional; e as de entrada: alavancagem, imobilização e custo operacional. Os resultados mostraram que a eficiência estava mais correlacionada com baixos custos e altas taxas de rentabilidade, o que quer dizer que neste setor, durante o período de análise, a competição esteve focada na redução do nível de imobilização, que ainda merece atenção de várias instituições que ainda não chegaram onde deveriam em relação a esta variável.

Lozano-Vivas et al. (2001) investigaram o desempenho das instituições bancárias na União Européia, avaliando, primeiramente, a eficiência das mesmas em cada país e posteriormente, após a inclusão das variáveis ambientais, a eficiência global em toda a Europa. $O$ estudo utilizou a modelagem DEA em mais de 600 bancos de 10 países. Como inputs foram utilizadas as variáveis: volume de mão-de-obra e estrutura física. E como outputs: volumes de empréstimos, depósitos e outros ativos.

Macedo et al. (2004) buscou, em seu estudo, através da utilização da DEA um índice - Índice de Performance Empresarial (IPE) - capaz de conjugar indicadores financeiros e não financeiros na análise de desempenho organizacional. Para tanto, utilizou uma amostra com os 17 maiores bancos brasileiros em operação em 2002, tendo como variáveis: imagem corporativa, riqueza gerada e rentabilidade do patrimônio líquido. Os autores concluíram que o modelo utilizado que foi desenvolvido a partir da DEA, foi capaz de conjugar em um único índice, indicadores de natureza diferente.

Camargo Jr. e Matias (2005) compararam, em seu estudo, as eficiências operacionais de 61 dos maiores bancos do Brasil, Argentina, Chile, Colômbia, EUA, México e Venezuela, utilizando DEA, orientação CRS. Foram considerados como inputs: ativo total, despesas com intermediação financeira, títulos mobiliários, exigível de longo prazo, depósitos totais e patrimônio líquido. E como outputs: receitas com intermediações financeiras e lucro antes do imposto de renda. Os resultados 
apontaram os bancos da Argentina e da Colômbia como os de menor eficiência.

No mesmo trabalho, posteriormente, os bancos foram segmentados em clusters obtidos com base nas variáveis contábeis utilizadas na pesquisa. Com isso, os autores conseguiram desenvolver um modelo discriminante de bom poder de previsão e classificação de um banco qualquer em um dos três clusters contábeis obtidos. Os poucos bancos da amostra de grandes valores para LAIR: ativo total, patrimônio líquido e depósitos totais, apresentaram índices intermediários de eficiência operacional. Em contrapartida, os bancos que apresentaram os maiores índices de eficiência mostraram também os menores valores para as variáveis LAIR.

Souza et al. (2003) avaliaram medidas de eficiência orientadas à produto para bancos brasileiros no ano de 2001, através da DEA - VRS. Nessa análise, produtos bancários, como títulos de investimento, empréstimos totais e depósitos à vista, foram analisados conjuntamente em uma medida única e como um vetor de produto múltiplo, para gerar diferentes medidas de eficiência da DEA, baseadas nos inputs: trabalho, fundos para empréstimo e estoque físico de capital. Foi utilizada a abordagem de intermediação e diversos modelos estatísticos foram considerados para cada medida eficiência, como ferramentas de modelagem para avaliar o significado dos efeitos técnicos: natureza do banco; tipo do banco; tamanho do banco; controle do banco; origem do banco e empréstimos inadimplentes. Os resultados mostraram que os bancos domésticos superaram, em termos de desempenho, os bancos estrangeiros e que os bancos de varejo apresentaram uma performance inferior à de todas as outras categorias de bancos. Além disso, nenhum dos modelos estudados mostra associação significante com os empréstimos inadimplentes (proxy para risco).

Seguindo a mesma linha de Souza et al. (2003), Sufian (2007) analisou a eficiência relativa, utilizando a DEA entre os bancos islâmicos domésticos e estrangeiros em operação na Malásia, entre os anos de 2002 e 2004. Os resultados mostraram também serem os bancos domésticos mais eficientes do que os estrangeiros.

Sanjeev (2006), em seu estudo, procurou descobrir se havia alguma relação 
entre o porte dos bancos e o desempenho alcançado por eles. Para isso, o autor submeteu sua amostra - bancos que operam no setor público indiano - à metodologia DEA - CRS, orientação input nos anos de 1997 a 2001. Testes complementares foram realizados e mostram não existir relação conclusiva entre o tamanho e o desempenho de instituições bancárias. Outros trabalhos também fizeram este questionamento, porém encontraram respostas diferentes.

Camargo Jr. et al. (2004) investigaram o desempenho de 19 bancos comerciais e múltiplos de grande porte em atuação no Brasil no ano de 2003, sob a abordagem DEA, considerando como inputs o ativo total, as despesas de pessoal e outras despesas administrativas, e como outputs, as operações de crédito, as operações de crédito de longo prazo, as aplicações em tesouraria e a rentabilidade da atividade bancária. Os principais resultados obtidos neste trabalho mostraram que os bancos mais eficientes eram aqueles com menor ativo total, isto é, os bancos menores. Além disso, os bancos mais eficientes também eram aqueles que conseguiam realizar grandes operações de concessão de crédito de longo prazo.

Souza e Macedo (2007) avaliaram, através da DEA, o desempenho de bancos de varejo no Brasil no período de 2001-2005, a partir de informações sobre rentabilidade, imobilização, custos, alavancagem, nível de depósito e de operações de crédito. Os autores concluíram o mesmo que Camargo Jr. et al. (2004), ou seja, que os melhores desempenhos estiveram associados a bancos de pequeno porte, quando comparados com os outros da amostra. Verificaram, ainda, neste trabalho, que das quatro instituições tidas como mais eficientes, apenas uma era de capital privado. Além disso, observou-se que os bancos de varejo eram mais eficientes no uso de seus custos e infraestrutura para captação de depósitos e operações de crédito, do que na transformação destes últimos em níveis ótimos de rentabilidade, com o uso adequado de níveis de alavancagem.

Leong et al. (2003) estão em consonância com a afirmação dos autores anteriores no que diz respeito a tamanho e desempenho. Com seu estudo sobre os bancos de Singapura, os pesquisadores destacaram terem potencial para uma maior 
ineficiência os maiores bancos e de mais complexas operações. Em contrapartida, Halkos e Salamouris (2004), ao avaliarem a eficiência de bancos gregos, concluíram que quanto maior $o$ ativo total, maior a eficiência.

\section{METODOLOGIA}

Com o objetivo de retratar a realidade do sistema bancário nacional, utilizou-se para a seleção da amostra deste estudo as instituições que se mantiveram classificadas, no período compreendido entre 2001 e 2007, no Relatório dos 50 Maiores Bancos, disponível no site do Banco Central. Desta publicação foi extraída não só a listagem dos bancos, como os demais dados necessários para a condução da pesquisa.

Das 50 instituições, apenas 28 se mantiveram classificadas como maiores no período sob análise. Dessas 28, 25 foram estudadas em profundidade, visto a ocorrência de problemas com as informações de três delas.

Após a definição da amostra a ser utilizada, julgou-se importante, a fim de que um perfil dos maiores e melhores bancos deste período fosse traçado, segmentar as instituições bancárias segundo alguns critérios: Ramo de Atividade, Controle de Capital e Porte. Isso ajudou a homogeneizar a amostra, fortalecendo, assim, a modelagem. A seguir, podem ser observados os critérios de segmentação.

Optou-se por considerar a divisão por ramo de atividade adotada pela revista Balanço Financeiro, da Gazeta Mercantil (Varejo, Atacado e Negócios, Financiamento e Middle Market), por considerá-la adequada às diferentes atividades, em virtude do foco do negócio exercido pelas instituições bancárias pesquisadas. Nos quadros a seguir, podem ser observados os critérios utilizados na classificação dos bancos nos segmentos, juntamente com as instituições em seus respectivos grupos. 
O sistema bancário brasileiro: Uma análise do desempenho através da DEA Ana Carolina Thomaz de Almeida Monteiro Barbosa, Marcelo Alvaro da Silva Macedo

\begin{tabular}{|c|l|}
\hline SEGMENTO & \multicolumn{1}{c|}{ DEFINIÇÃO } \\
\hline Varejo & Esta categoria inclui os bancos privados com foco no varejo e que possuem mais de 200 agências. \\
\hline Financiamento & Os bancos desta categoria têm como atividade principal oferecer crédito para o financiamento de compras no varejo. \\
\hline Atacado e Negócios & $\begin{array}{l}\text { Os bancos de atacado e negócio possuem, individualmente, um número reduzido de clientes e estão focadas em } \\
\text { pessoas físicas de alta renda e empresas. }\end{array}$ \\
\hline Middle Market & Esta categoria compõe-se de instituições voltadas para o mercado de empresas de pequeno e médio porte \\
\hline
\end{tabular}

Quadro 01: Base de Segmentação por Ramo

\begin{tabular}{l|l|l|l}
\hline BANCO & CLASSIFICAÇÃO & BANCO & CLASSIFICAÇÃ O \\
\hline BB & Varejo & BANRISUL & Varejo \\
\hline ITAU & Varejo & BBM & Atacado e Negócios \\
\hline BRADESCO & Varejo & DEUTSCHE & Atacado e Negócios \\
\hline CEF & Varejo & BIC & Middle Market \\
\hline ABN AMRO & Varejo & ING & Atacado e Negócios \\
\hline UNIBANCO & Varejo & BASA & Varejo \\
\hline SANTANDER & Varejo & BANESTES & Varejo \\
\hline HSBC & Varejo & SS & Varejo \\
\hline VOTRA & Atacado e Negócios & MERCANTIL DO BRASIL & Varejo \\
\hline CITIBANK & Atacado e Negócios & RABOBANK & Atacado e Negócios \\
\hline NOSSA CAIXA & Atacado e Negócios & BRB & Varejo \\
\hline UBS PACTUAL & Atacado e Negócios & RURAL & Middle Market \\
\hline
\end{tabular}

Quadro 02: Classificação das Instituições Bancárias por ramo

A partir de informações do relatório do BC, as instituições bancárias da amostra também foram classificadas com relação ao tipo de controle de capital.

\begin{tabular}{|l|l|l|l|}
\hline BANCO & CLASSIFICAÇÃO & BANCO & CLASSIFICAÇÃO \\
\hline BB & Público Federal & BANRISUL & Público Estadual \\
\hline ITAU & Privado Nacional & BBM & Privado Nacional \\
\hline BRADESCO & Privado Nacional & DEUTSCHE & Privado Controle Estrangeiro \\
\hline CEF & Público Federal & BIC & Privado Nacional \\
\hline ABN AMRO & Privado Controle Estrangeiro & ING & Privado Controle Estrangeiro \\
\hline UNIBANCO & Privado Participação Estrangeira & BASA & Público Federal \\
\hline SANTANDER & Privado Controle Estrangeiro & BANESTES & Público Estadual \\
\hline HSBC & Privado Controle Estrangeiro & SS & Privado Nacional \\
\hline SAFRA & Privado Nacional & MERCANTIL DO BRASIL & Privado Nacional \\
\hline VOTORANTIM & Privado Nacional & RABOBANK & Privado Controle Estrangeiro \\
\hline CITIBANK & Privado Controle Estrangeiro & BRB & Público Estadual \\
\hline NOSSA CAIXA & Público Estadual & RURAL & Privado Nacional \\
\hline UBS PACTUAL & Privado Controle Estrangeiro & & \\
\cline { 1 - 2 } & & &
\end{tabular}

Quadro 03 - Classificação das Instituições Bancárias por Controle de Capital

A classificação das instituições por porte foi feita, assim como no trabalho de Paula e Faria (2007), a partir de critério do BC, com base no ativo total ajustado apresentado pelos bancos. As instituições cujo percentual de participação individual é 
superior a $15 \%$ são consideradas de grande porte e excluídas da amostra; A nova amostra é então reclassificada em ordem decrescente das participações individuais das empresas no total dos ativos dessa amostra. As instituições que compõe a faixa de até $70 \%$, inclusive, do montante de participação acumulada, também são consideradas de grande porte, juntamente com aquelas apuradas no início e excluídas. As instituições que compõem a faixa entre $70 \%$ e $95 \%$ são consideradas de médio porte e as que compõem a faixa acima de $95 \%$ são consideradas de pequeno porte.

\begin{tabular}{|l|l|l|l|}
\hline \multicolumn{1}{|c|}{ BANCO } & \multicolumn{1}{|c|}{ CLASSIFICAÇÂO } & \multicolumn{1}{c|}{ BANCO } & Médio Porte \\
\hline BB & Grande Porte & BANRISUL & Médio Porte \\
\hline ITAU & Grande Porte & BBM & Médio Porte \\
\hline BRADESCO & Grande Porte & DEUTSCHE & Médio Porte \\
\hline CEF & Grande Porte & BIC & Pequeno porte \\
\hline ABN AMRO & Grande Porte & ING & Médio Porte \\
\hline UNIBANCO & Grande Porte & BANA & Médio Porte \\
\hline SANTANDER & Grande Porte & SS & Médio Porte \\
\hline SAFRA & Pequeno Porte & MERCANTIL DO BRASIL & Médio Porte \\
\hline VOTORANTIM & Médio Porte & RABOBANK & Médio Porte \\
\hline CITIBANK & Médio Porte & BRB & Pequeno porte \\
\hline NOSSA CAIXA & Médio Porte & RURAL & Pequeno porte \\
\hline UBS PACTUAL & Médio Porte & & \\
\hline
\end{tabular}

Quadro 04 - Classificação das Instituições Bancárias por Porte

De cada instituição selecionada foram coletadas informações referentes às contas das demonstrações contábeis de maior relevância para o setor, conforme apontava o relatório, disponíveis no período analisado. São elas: Imobilização, Número de Agências, Número de Funcionários e Lucro Líquido.

A partir destas contas foram criados os indicadores deste estudo, apresentados a seguir:

- Número de Agências: Mostra o volume médio de agências no período 20012007. É um indicador do tipo quanto menor, melhor;

- Número de Funcionários: Mostra o volume médio de funcionários no período 2001-2007. É um indicador do tipo quanto menor, melhor;

- Grau de Imobilização: Mostra o percentual médio de Imobilização (parcela do 
Patrimônio Líquido comprometida com ativos permanentes) no período 20012007. É um indicador do tipo quanto menor, melhor;

- Lucro Líquido: Mostra o volume total de lucro no período 2001-2007. É um indicador do tipo quanto maior, melhor.

Em suma, no modelo de análise proposto verifica-se o desempenho como função do sacrifício médio do período como input, medido pelo número médio de funcionários e agências e pelo grau médio de imobilização, e do benefício total do período, medido pelo somatório dos lucros anuais.

Um grande número de diferentes variáveis, como as apresentadas anteriormente, pode ser utilizado para avaliar a eficiência de unidades organizacionais como bancos, onde cada uma destas pode ser vista como um vetor de desempenho isolado, representando um diferente aspecto da performance. Estes seriam modelos de avaliação de performance monocriteriais.

O que se busca neste artigo é apresentar uma metodologia multidimensional, na qual seja possível avaliar o desempenho de cada instituição bancária de modo multicriterial, ou seja, utilizando de maneira integrada todos os vetores de desempenho apresentados. Entretanto, é importante destacar que a modelagem não se limita à avaliação de desempenho, fornecendo também os pontos ótimos que as instituições bancárias deveriam atingir em relação aos parâmetros determinados. Essa análise recebe o nome de benchmarking, se caracterizando por procurar propor, com base no que as unidades eficientes já conseguem atingir, valores ideais para os indicadores tidos como não eficientes.

Isto é feito através da utilização da DEA, que mostra o quão um banco é eficiente no tratamento de seus inputs e outputs, em relação aos outros, numa análise que fornece um indicador que varia de 0 a 1 ou de $0 \%$ a $100 \%$. Somente as instituições que obtêm índice de eficiência igual a um é que são efetivamente eficientes, ou seja, fazem parte da fronteira eficiente.

Em função das características do setor, optou-se pela utilização tanto do método 
DEA CRS-O, quanto DEA CRS-I, através de dois modelos criados com objetivo de melhor caracterização do segmento. Os modelos foram executados em um software de DEA, apresentado por Meza et al. (2003), denominado SIAD (Sistema Integrado de Apoio à Decisão), tendo como inputs: Média de Funcionários, Média de Agências e Média do Grau de Imobilização. E como output: LL do período 2001-2007.

\section{APRESENTAÇÃO E ANÁLISE DOS RESULTADOS}

Após o tratamento dos dados, por modelo, no SIAD, foi feita uma análise da eficiência dos bancos numa amplitude de 0 a 100\%. Estas eficiências são apresentadas na tabela a seguir.

Tabela 1 - Eficiência dos Bancos Sob Análise

\begin{tabular}{|l|r|l|r|}
\hline \multicolumn{1}{|c|}{ Bancos } & Desempenho & Bancos & Desempenho \\
\hline BB & $69,51 \%$ & BANRISUL & $20,74 \%$ \\
\hline ITAU & $100,00 \%$ & BBM & $42,29 \%$ \\
\hline BRADESCO & $84,33 \%$ & DEUTSCHE & $36,61 \%$ \\
\hline CEF & $34,53 \%$ & BIC & $11,24 \%$ \\
\hline ABN AMRO & $47,88 \%$ & ING & $37,10 \%$ \\
\hline UNIBANCO & $73,35 \%$ & BASA & $23,46 \%$ \\
\hline SANTANDER & $72,14 \%$ & BANESTES & $7,14 \%$ \\
\hline HSBC & $26,46 \%$ & SS & $69,97 \%$ \\
\hline SAFRA & $76,37 \%$ & MERCANTIL DO BRASIL & $3,00 \%$ \\
\hline VOTORANTIM & $100,00 \%$ & RABOBANK & $15,17 \%$ \\
\hline CITIBANK & $82,01 \%$ & BRB & $6,37 \%$ \\
\hline NOSSA CAIXA & $19,52 \%$ & RURAL & $5,06 \%$ \\
\hline UBS PACTUAL & $95,67 \%$ & & \\
\cline { 1 - 2 } & &
\end{tabular}

Pode-se verificar, quanto ao desempenho, que apenas o VOTORANTIM e o ITAÚ foram eficientes. Estes foram seguidos por outros bancos com bons indicadores de desempenho (próximos a $100 \%$ ), tais como: BRADESCO e CITIBANK. Na verdade, estes bancos foram os que melhor combinaram os inputs (menores) e output (maior). Assim sendo, o VOTORANTIM e o ITAÚ não precisam melhorar em nada seu desempenho, enquanto que os outras precisam de pequenas alterações em seus inputs e/ou output para se tornarem eficientes. Os bancos MERCANTIL DO BRASIL, RURAL, BRB e BANESTES, que possuíam 
índices de eficiência baixíssimos, necessitam de mudanças radicais.

Para uma avaliação mais detalhada são utilizadas as maiores eficiências encontradas. Destas instituições, 50\% são do segmento Varejo e 50\% Atacado e Negócios, sendo todas as de Varejo de Grande Porte e as de Atacado e Negócios, Médio Porte. Observa-se também que $90 \%$ das instituições com melhores desempenhos são privadas, sendo $45 \%$ de controle puramente nacional. Das instituições menos eficientes, tem-se a maioria de médio porte. Sendo $71 \%$ de Varejo e o restante Middle Market.

Uma outra análise interessante gerada pelo uso da DEA é a de benchmarking. Pode-se perceber que o VOTORANTIM é o banco que mais aparece como benchmark para os outros em todas as análises. Isso se dá pelo fato de ser este o banco de destaque, em termos de desempenho multicriterial, em relação às variáveis estudadas no período analisado.

Os dados atuais e propostos pela metodologia, presentes nos quadros a seguir, são analisados nessa seção. Tanto o direcionamento input (tabela 3 ), quanto output (tabela 2) serão sugeridos como alternativas de melhoria às instituições bancárias. No primeiro, tem-se a redução dos inputs mantendo-se o nível de output. Já no segundo, tem-se o incremento do output, mantendo-se os inputs.

Tabela 2 - Valores Ideais x Valores Atuais / Orientação Output

\begin{tabular}{rrrrrrrr} 
ATUAL & IDEAL & \multicolumn{1}{c}{ ATUAL } & \multicolumn{1}{c}{ IDEAL } & ATUAL & IDEAL & ATUAL & IDEAL \\
$11.799 .108,0$ & $11.799 .108,0$ & $101.500,9$ & $101.501,0$ & $3.635,7$ & $3.636,0$ & 0,2352 & 0,2400 \\
$16.974 .072,0$ & $16.974 .072,0$ & $52.616,9$ & $52.617,0$ & $2.309,7$ & $2.310,0$ & 0,4520 & 0,4500 \\
$14.313 .586,0$ & $16.974 .072,0$ & $69.283,6$ & $52.617,0$ & $2.966,7$ & $2.310,0$ & 0,4543 & 0,4500 \\
$4.747 .783,0$ & $13.996 .928,4$ & $103.516,3$ & $52.220,7$ & $2.163,1$ & $2.163,0$ & 0,3538 & 0,3500 \\
$4.375 .897,0$ & $9.139 .295,1$ & $28.545,3$ & $23.829,2$ & $1.038,4$ & $1.038,0$ & 0,3041 & 0,2071 \\
$6.175 .491,0$ & $8.418 .643,5$ & $24.998,6$ & $21.181,2$ & 920,6 & 921,0 & 0,4589 & 0,1847 \\
$6.150 .203,0$ & $8.525 .266,2$ & $21.573,3$ & $21.573,0$ & $1.035,9$ & 938,3 & 0,2567 & 0,1880 \\
$2.261 .628,0$ & $8.547 .991,2$ & $24.165,9$ & $21.656,5$ & 941,9 & 942,0 & 0,3832 & 0,1888 \\
$2.534 .554,0$ & $3.318 .647,2$ & $4.774,0$ & $2.442,0$ & 93,4 & 93,0 & 0,1761 & 0,0266 \\
$2.782 .778,0$ & $2.782 .778,0$ & 473,1 & 473,0 & 6,3 & 6,0 & 0,0134 & 0,0100 \\
$2.605 .321,0$ & $3.176 .980,6$ & $3.247,0$ & $1.921,4$ & 70,4 & 70,0 & 0,1692 & 0,0222 \\
$1.156 .218,0$ & $5.924 .080,1$ & $14.680,0$ & $12.015,3$ & 516,0 & 516,0 & 0,2117 & 0,1074 \\
$1.775 .465,0$ & $1.855 .185,3$ & 441,9 & 315,3 & 3,9 & 4,0 & 0,0727 & 0,0067 \\
$1.067 .948,0$ & $5.147 .993,7$ & $9.874,9$ & $9.163,7$ & 389,6 & 390,0 & 0,2359 & 0,0833 \\
$671.731,0$ & $1.588 .477,9$ & 270,4 & 270,0 & 4,7 & 3,4 & 0,2262 & 0,0057 \\
$475.973,0$ & $1.300 .198,6$ & 220,9 & 221,0 & 2,9 & & &
\end{tabular}


Pode-se perceber que todas as variáveis apresentaram melhorias na situação proposta pela metodologia. A variável input - \% de Imobilização - totalmente relacionada à "produção" de receita e elevação dos índices de lucro da empresa (output) merece atenção especial, uma vez que a sua redução, a princípio, implica em aumento de LL, ao passo que se tem mais dinheiro descomprometido ou disponível para investimentos. Em contrapartida, o aumento de LL não impulsiona variações diretas (redução) no percentual de imobilização. Sendo assim, sugere-se um caminho híbrido, ou seja, tanto de redução desta variável, como de alternativas capazes de aumentar o lucro.

Por último, ainda com base nos resultados, procedeu-se uma análise adicional em relação às variáveis. Foi feita uma verificação dos pesos atribuídos aos vetores de desempenho, para saber, com base em pesos iguais a zero, as variáveis que estavam sendo "desprezadas" na análise de desempenho.

O indicador de Imobilização foi o que apresentou o maior número de zeros nos pesos da modelagem DEA. Isso significa que este é o indicador mais "desprezado" na análise de desempenho. Em linhas gerais, quando uma variável tem peso ( $u$ ou v) igual a zero, isto representa que estas variáveis são naturalmente problemáticas na obtenção dos índices de eficiência. Como a modelagem procura os melhores índices de desempenho, dadas às características de inputs e outputs, para cada DMU, esta atribui zero a toda variável que possa atrapalhar o objetivo de maximizar a eficiência.

Em termos gerenciais isso quer dizer que o Grau de Imobilização, que é uma variável que já vem sendo reduzida pelos bancos ao longo dos anos, tem margem para reduções ainda maiores, pois a grande presença de pesos zero pode ser atribuída a dispersão desta variável, que possui desde instituições com baixos valores $(1,34 \%)$ até outras com alto grau de imobilização (46,70 \%).

\section{CONCLUSÃO}

A eficiência é um dos aspectos que vêm governando, em maior ou menor grau, 
dependendo da organização, a atuação de bancos e outras instituições financeiras no Brasil e no mundo, nos últimos anos, conforme é mostrado nos estudos referendados nesta pesquisa.

Numa análise dos resultados apresentados, pode-se perceber que as melhores instituições bancárias são aquelas que possuem um desempenho multicriterial superior. Por outro lado, as tidas como ineficientes pela modelagem, precisam de mudanças drásticas em seus inputs e outputs.

Além disso, pode-se perceber ser o segmento Atacado e Negócios o que mais apresentou instituições eficientes, seguido pelo Varejo. Já o Middle Market foi ressaltado apenas no grupo das ineficientes, ficando o Financiamento com a pior classificação. Isto aconteceu porque nenhuma instituição deste grupo apareceu no ranking do $\mathrm{BC}$, no período analisado.

Quanto ao porte, não se observou relação direta entre tamanho e desempenho. Entretanto, percebeu-se que as instituições mais eficientes, ou tinham controle totalmente estrangeiro ou, pelo menos, parcial.

Por fim, os resultados desse estudo como um todo, propõem uma nova percepção sobre a performance financeira de instituições bancárias que não se encontram disponíveis aos gestores e ao mercado em geral através dos balanços e tradicionais análises de índices financeiros. Ou seja, a partir de informações que não estariam disponíveis pelas técnicas convencionais, os resultados da Análise Envoltória de Dados podem proporcionar melhores condições de competitividade às instituições bancárias, principalmente quando interpretadas e usadas com os conhecimentos e julgamentos próprios da alta administração sobre suas operações.

O assunto não se encontra encerrado, pois ainda há muito a ser explorado desta metodologia em gestão de instituições financeiras. Este mesmo trabalho deve ter continuidade através de uma melhor visão dos vetores de desempenho que mais contribuem para uma mensuração mais apurada da eficiência organizacional, aplicando a metodologia aqui apresentada e discutida combinada a outras metodologias. 
O sistema bancário brasileiro: Uma análise do desempenho através da DEA Ana Carolina Thomaz de Almeida Monteiro Barbosa, Marcelo Alvaro da Silva Macedo

\section{REFERÊNCIAS}

BANKER, R.D.; CHARNES, A. e COOPER, W.W. 1984. Some Models for Estimating Technical and Scale Inefficiencies in Data Envelopment Analysis. Management Science, 30(9): 1078-1092.

BARR, R.S.; KILLGO, K.A.; SIEMS, T.F. e ZIMMEL, S. 2002. Evaluating the Productive Efficiency and Performance of U.S. Commercial Banks. Managerial Finance, 28(8):3-25.

CAMARGO Jr. A.S.; MATIAS, A.B. e MARQUES, F.T. 2004. Desempenho dos Bancos Comerciais e Múltiplos de Grande Porte no Brasil. In: Congresso Latinoamericano de Escolas De Administração, 39, 2004, San Domingo, República Dominicana. Anais... XXXIX CLADEA. San Domingo/República Dominicana: CLADEA, 1 CD.

CAMARGO Jr. A.S. e MATIAS, A.B. 2005. Eficiência operacional de bancos no Brasil, América Latina e EUA. In: Congresso Internacional De Custos, 9, 2005, Itapema/SC. Anais ... do IX Congresso do IIC. Itapema/SC: IIC, 1 CD.

CAMP, R.C. 1989. Benchmarking: the search for industry best practices that lead to superior performance. New York: Quality Resources. 299 p.

CHARNES, A.; COOPER, W.W. e RHODES, E. 1978. Measuring the Efficiency of Decision Making Units. European Journal of Operational Research, 2(6): 429444.

CHARNES, A.; COOPER, W.W.; LEWIN, A.Y. e SEIFORD, L.M. 1994. Data Envelopment Analysis. 2. ed. Boston: KAP. 510p.

COELLI, T.; RAO, D.S.P. e BATTESE, G.E. 1998. An Introduction to Efficiency and Productivity Analysis. Boston: KAP. 275 p.

FARREL, M.J. 1957. The Measurement of Productive Efficiency. Journal of the Royal Statistical Society, 120(3)A: 253-290.

HALKOS, G.E. e SALAMOURIS, D.S. 2004. Efficiency measurement of the Greek commercial banks with the use of financial ratios: A data envelopment analysis approach. Management Accounting Research, 15(2):201-224.

LEONG, W.H.; DOLLERY, B. e COELLI, T. 2003. Measuring the technical efficiency of banks in Singapure for the period 1993 to 1999: an application and extension of the Bauer Et Al (1997) Technique. ASEAN Economic Bulletin, 20(3): 195-210. 
O sistema bancário brasileiro: Uma análise do desempenho através da DEA Ana Carolina Thomaz de Almeida Monteiro Barbosa, Marcelo Alvaro da Silva Macedo

MEZA, L.A.; BIONDI NETO, L; SOARES DE MELLO, J.C.C.B.; GOMES. E.G. e COELHO, P.H.G. 2003.

SIAD - Sistema Integrado de Apoio à Decisão: uma implementação computacional de modelos de análise de envoltória de dados. In: Simpósio de Pesquisa Operacional da Marinha, 6, 2003, Rio de Janeiro. Anais... VI SPOLM. Rio de Janeiro: CASNAV, 1 CD.

MOSTAFA, M. 2007. Benchmarking top Arab banks' efficiency through efficient frontier analysis (DEA). Industrial Management \& Data Systems, 107(6):802-823. NEELY, A. 1998. Measuring business performance. London: The Economist Books. 208 p.

PAULA, L.F. e FARIA, J.A. 2007. Eficiência no setor bancário brasileiro por segmento de mercado: Uma avaliação recente. In: Encontro Nacional de Economia, 35, 2007, Recife. Anais... XXXV ANPEC. Recife: ANPEC, 1 CD.

REVISTA BALANÇO FINANCEIRO. São Paulo: Gazeta Mercantil, 2007. Anual. Ranking Completo de 418 Instituições. 122 p.

Relatório dos 50 Maiores Bancos. Disponível em://http www.bcb.gov.br, acesso em 15/04/2008. SANJEEV, G.M. 2006. Does banks' size matter in India? Journal of Services Research, 6(2):135-144.

SILVA, A.C. Magalhães da; NEVES, C.; GONÇALVES NETO, A.C. 2003. Avaliação da Eficiência das Companhias de Seguro no ano de 2002: uma abordagem através da Análise Envoltória de Dados. In: Congresso Brasileiro de Custos, 10, 2003, Guarapari/ES. Anais... X Congresso Brasileiro de Custos. Guarapari: ABC, 1 CD.

SLACK, N.; CHAMBERS, S.; HARLAND, C.; HARRISON, A. e JOHNSTON, R. 2002. Administração da Produção. 2 ed. São Paulo: Atlas. 754 p.

SOUZA, M.F.A. e MACEDO, M.A.S. 2007. Desempenho em Bancos de Varejo no Brasil: uma discussão apoiada em Análise Envoltória de Dados (DEA). In: Congresso Internacional de Custos, 10, 2007, Lyon/França. Anais... X Congresso do IIC. Lyon/França: IIC, 1 CD.

SOUZA, G.S.; TABAK, B.M.; STAUB, R.B. 2003. Avaliação da Significância do Efeito de Fatores nas Medidas de Eficiência da DEA Orientada a Produto: Aplicação para Bancos Brasileiros. Relatório de Estabilidade Financeira, Banco Central do Brasil, 2(2):129-145.

SUFIAN, F. 2007. The efficiency of Islamic banking industry in Malaysia. Humanomics, 23(3):174-192. 
O sistema bancário brasileiro: Uma análise do desempenho através da DEA Ana Carolina Thomaz de Almeida Monteiro Barbosa, Marcelo Alvaro da Silva Macedo

Data de Submissão: 19/11/2008

Data de Aceite: 16/12/2008 